\title{
Transitive sets and cyclic quadrilaterals
}

\author{
Imre Leader, Paul A. Russell and Mark Walters
}

\begin{abstract}
Motivated by some questions in Euclidean Ramsey theory, our aim in this note is to show that there exists a cyclic quadrilateral that does not embed into any transitive set (in any dimension). We show that in fact this holds for almost all cyclic quadrilaterals, and we also give explicit examples of such cyclic quadrilaterals. These are the first explicit examples of spherical sets that do not embed into transitive sets.
\end{abstract}

KEYWORdS AND PHRASEs: Ramsey theory, Euclidean Ramsey theory, transitive sets.

\section{Introduction}

A finite set $X$ in some Euclidean space $\mathbb{R}^{n}$ is called Ramsey if for every positive integer $k$ there exists $d$ such that whenever $\mathbb{R}^{d}$ is $k$-coloured it contains a monochromatic set congruent to $X$. A famous question of Erdős, Graham, Montgomery, Rothschild, Spencer and Straus [1] (made into a conjecture by Graham in [3]) asks if the Ramsey sets are precisely the spherical sets (where a set is spherical if it lies on the surface of a sphere).

In [4], a "rival" conjecture is made: that a set is Ramsey if and only if it is a subset of a finite transitive set (in some dimension) - here we say that a set is transitive if its isometry group acts transitively on it (or equivalently, loosely speaking, if all points of the set look the same). It is not obvious that these conjectures are different: certainly every finite transitive set $X$ is spherical (for example, because all of its points must lie on the surface of the minimal sphere containing $X$ ), but does every finite spherical set embed into a transitive set (in some dimension)? This is answered in the negative in [4], where it is shown that, for $k \geq 16$, almost every cyclic $k$-gon does not embed into a (finite) transitive set.

However, in [4] we conjectured that it was unnecessary to go as far as 16-gons to see this phenomenon: that in fact there exists a cyclic quadrilateral that does not embed into a transitive set. (This would be the smallest

arXiv: 1012.5468 
possible case, as all triangles do embed into transitive sets - see e.g. [2] or [4]).

Our aim in this note is to prove this. In fact, we show that almost every cyclic quadrilateral does not embed into a transitive set. Our proof allows us to give an explicit example of such a cyclic quadrilateral. This is the first explicit example of a spherical set that does not embed into a transitive set.

Curiously, it seems that the right approach is to focus on the linear properties of the quadrilateral, rather than its metric properties. Our main result is as follows.

Theorem 1. Let $x, y, z$ and $w$ be four distinct points lying on a circle such that

$$
w=z+\alpha(x-z)+\beta(y-z),
$$

where $\alpha \neq 1$ and $\beta$ is transcendental over $\mathbb{Q}(\alpha)$. Then xyzw does not embed into a transitive set.

We remark that the condition $\alpha \neq 1$ is necessary. Indeed, for any $\beta$ there is a trapezium with parameters 1 and $\beta$ (in other words, with this value of $\beta$ and with $\alpha=1$ ) which embeds in a transitive set with symmetry group $D_{8}$. We note also that the "cyclic" condition is trivially redundant as all quadrilaterals that embed in a transitive set are cyclic.

Since "many" pairs $(\alpha, \beta)$ can occur as parameters of cyclic quadrilaterals (for example, any pair $(\alpha, \beta)$ with $\alpha$ and $\beta$ sufficiently close to 1 ) it is routine to verify from this that almost every cyclic quadrilateral does not embed into a transitive set. It is also possible to give an explicit example even one with some symmetry, such as a kite.

Corollary 2. The cyclic quadrilateral with vertices

$$
(-1,0),(1,0),\left(a, \sqrt{1-a^{2}}\right),\left(a,-\sqrt{1-a^{2}}\right),
$$

where $a$ is transcendental, does not embed into any transitive set.

Corollary 2 follows from Theorem 1 upon taking $z=(-1,0), y=(1,0)$, $x=\left(a, \sqrt{1-a^{2}}\right)$ and $w=\left(a,-\sqrt{1-a^{2}}\right)$.

As explained above, this gives us an explicit spherical set that we conjecture is not Ramsey.

Conjecture 3. Let $-1<a<1$ be transcendental. Then the cyclic quadrilateral with vertices

$$
(-1,0),(1,0),\left(a, \sqrt{1-a^{2}}\right),\left(a,-\sqrt{1-a^{2}}\right)
$$

is not Ramsey. 


\section{Proof of Theorem 1}

It will be convenient to use the term quadrilateral to denote any set of four coplanar points, whether or not they are distinct. We say that it is trivial if all four points are coincident. Note that a non-trivial quadrilateral may still have some points coincident.

Suppose that $x y z w$ is any quadrilateral. It may be the case (for example whenever $x, y, z$ are not collinear) that there exist (not necessarily unique) $\alpha, \beta$ such that $w=z+\alpha(x-z)+\beta(y-z)$. In this case we say that the quadrilateral has parameters $\alpha, \beta$.

One reason why this parameterisation in terms of linear rather than metric properties is useful is as follows. Suppose we have a non-trivial quadrilateral in a vector space $V \oplus W$. Then the projections onto $V$ and $W$ have the same parameters. Moreover at least one of these projections is non-trivial. (Note that both projections may have coincident points even if the original quadrilateral does not, for example if the original quadrilateral is a rectangle.) This will allow us to focus on irreducible representations.

The following is our key result.

Lemma 4. Let $G$ be a finite group generated by elements $g, h$ and $k$, and let $A, B$ and $C$ be the maps corresponding to $g, h$ and $k$ respectively in a nontrivial irreducible real orthogonal representation of $G$. Then the collection of pairs $(\alpha, \beta)$ that can occur in quadrilaterals of the form $A(w), B(w), C(w), w$ for some $w \neq 0$ is exactly the zero set of a polynomial $P(\alpha, \beta)$ with algebraic coefficients. Moreover, for any fixed $\alpha \neq 0,1$ the polynomial $P$ viewed as a polynomial in $\beta$ is not identically zero.

Proof. Let $d$ be the dimension of the representation. Suppose that there exists non-zero $w \in \mathbb{R}^{d}$ with

$$
w=C(w)+\alpha(A(w)-C(w))+\beta(B(w)-C(w)) .
$$

Then

$$
(\alpha A+\beta B+(1-\alpha-\beta) C-I)(w)=0
$$

and so, in particular, the linear map

$$
L(\alpha, \beta)=\alpha A+\beta B+(1-\alpha-\beta) C-I
$$

is singular. Conversely, if $L(\alpha, \beta)$ is singular then there exists non-zero $w$ with $L(\alpha, \beta)(w)=0$; that is, with $w$ satisfying (1). 
Obviously $P(\alpha, \beta)=\operatorname{det}(L(\alpha, \beta))$ is a polynomial in $\alpha$ and $\beta$. It is well known that any representation of $G$ over $\mathbb{R}$ is equivalent to a representation with all matrix entries algebraic. Since the polynomial $\operatorname{det}(L(\alpha, \beta))$ is the same for equivalent representations, this implies that $P(\alpha, \beta)$ has algebraic coefficients.

Suppose $\alpha \neq 0,1$. Since $P$ is a polynomial, to complete the proof we just need to give one value of $\beta$ for which $P$ is non-zero or, equivalently, for which there exists non-zero $w$ such that $L(\alpha, \beta)(w)=0$. This is the same as saying that there is no non-zero $w$ for which the quadrilateral $w, A(w)$, $B(w), C(w)$ has parameters $\alpha$ and $\beta$.

We note first that a quadrilateral $w, A(w), B(w), C(w)$ cannot be trivial; that is

$$
w=A(w)=B(w)=C(w)
$$

cannot occur for any (non-zero) $w$. Indeed, this would imply that the representation is either reducible or one-dimensional. The former cannot occur by hypothesis; the latter cannot occur since then $A=B=C=I$, which is ruled out as the representation is non-trivial.

Rather surprisingly, we will be able to choose $\beta$ in such a way that the only possible (non-trivial) quadrilaterals with parameters $\alpha$ and $\beta$ that occur in this way are not even convex (so manifestly are not cyclic and so do not embed in a transitive set). The proof splits into three cases: $\alpha<0$, $0<\alpha<1$, and $\alpha>1$.

First, if $0<\alpha<1$ then let $\beta=(1-\alpha) / 2$. Then (1) becomes

$$
w=\alpha A(w)+\frac{1-\alpha}{2} B(w)+\frac{1-\alpha}{2} C(w) .
$$

Since $\alpha>0$ and $(1-\alpha) / 2>0$, this implies (2), a contradiction.

Secondly, if $\alpha<0$ then let $\beta=\alpha$. Then (1) becomes

$$
(-\alpha) A(w)+(-\alpha) B(w)+w=(1-2 \alpha) C(w)
$$

Since $-\alpha$ and $1-2 \alpha$ are both positive, once again this implies (2).

Finally, if $\alpha>1$ then let $\beta=(1-\alpha) / 2$. By a similar argument to the previous case, this again implies (2). This completes the proof.

Proof of Theorem 1. Suppose that we have a transitive set $T$ with symmetry group $G$ and a non-trivial quadrilateral $x y z w$ in $T$ with

$$
w=z+\alpha(x-z)+\beta(y-z) .
$$


We aim to show that $\beta$ is algebraic over $\mathbb{Q}(\alpha)$. Let $A, B, C$ be elements of $G$ that map $w$ to $x, y, z$ respectively, and let $G^{\prime}$ be the group generated by $A, B$ and $C$.

We shall use Lemma 4, but first observe that if $\alpha=0$ then $w, z$ and $y$ are collinear and so at least two must coincide, contradicting the hypothesis of the theorem.

Suppose that the representation of $G^{\prime}$ is reducible as $V \oplus W$. Then (3) holds for the projections onto $V$ and $W$, and in at least one of these cases the projected quadrilateral is non-trivial.

It follows that the parameters $(\alpha, \beta)$ occur for some (non-trivial) irreducible representation of $G^{\prime}$. This implies that $P(\alpha, \beta)=0$ for some polynomial $P$ as in the conclusion of Lemma 4. Writing $R(Y)$ for $P(\alpha, Y)$ and $\mathbb{A}$ for the field of algebraic numbers, we have that the polynomial $R$ has coefficients in $\mathbb{A}(\alpha)$ and is not identically zero. Moreover, $R(\beta)=0$. Hence $\beta$ is algebraic over $\mathbb{A}(\alpha)$ and so also over $\mathbb{Q}(\alpha)$.

\section{References}

[1] Erdős, P., Graham, R. L., Montgomery, P., Rothschild, B. L., Spencer, J., and Straus, E. G. (1973). Euclidean Ramsey theorems. I., J. Comb. Theory Ser. A 14 341-363. MR0316277

[2] Frankl, P., and Rödl, V (1986). All triangles are Ramsey, Trans. Amer. Math. Soc. 297 777-779. MR0854099

[3] Graham, R. L. (1994). Recent trends in Euclidean Ramsey theory, Discrete Math. 136 119-127. MR1313284

[4] Leader, I., Russell, P. A., and Walters, M. (2012). Transitive sets in Euclidean Ramsey theory. J. Comb. Theory Ser. A 119 382-396.

IMRE LEADER,

Department of Pure Mathematics and Mathematical Statistics

Centre for Mathematical Sciences

Wilberforce Road, Cambridge CB3 0WB

ENGLAND

E-mail address: I.Leader@dpmms.cam.ac.uk

Paul A. Russell

Department of Pure Mathematics and Mathematical Statistics

Centre for Mathematical Sciences

Wilberforce Road, Cambridge CB3 0WB

ENGLAND

E-mail address: P.A.Russell@dpmms.cam.ac.uk 
Mark Walters

School of Mathematical Sciences QUEen Mary, University OF LONDON LONDON E1 4NS

ENGLAND

E-mail address: m.walters@qmul.ac.uk

Received December 29, 2010 